\title{
Hypertrophe Kardiomyopathie
}

\section{Wie riskant ist der Gendefekt?}

Die hypertrophe Kardiomyopathie (HCM) wird oft zufällig entdeckt. Doch wie sieht dann das diagnostische und therapeutische Prozedere aus?

Pathogenetisch liegt bei der hypertrophen Kardiomyopathie (HCM) eine Störung im Bereich der Sarkomere vor; in etwa $60 \%$ eine autosomal dominante sarkomerische Mutation. Bei einem Drittel bleibt die Ursache jedoch unklar; bei wenigen Patienten ist die HCM Teil einer syndromalen Erkrankung wie der Friedreichen Atrophie oder Muskeldystrophie.

„Immer wenn muskuläre oder neurologische Symptome vorliegen, sollte man auch an eine hypertrophe Kardiomyopathie denken", erläuterte Professor Norbert Frey, Universitätsklinik in Kiel. „,Red flags“" seien die EKG-Veränderungen, die aber meist unspezifisch sind. Am häufigsten sind Hypertrophie-Zeichen bzw. tiefe TNegativierungen in den BW-Ableitungen.

\section{Obstruktiv oder nicht obstruktiv?}

Das diagnostische Standardverfahren ist die Farbdoppler-Echo. Bei etwa 70\% der Patienten findet sich hier eine Obstruktion im linksventrikulären Ausflusstrakt. Bei einem Druckgradienten von in Ruhe min. $50 \mathrm{mmHg}$ ist die Diagnose hypertrophe obstruktive Kardiomyopathie (HOCM) gesichert. Sonst empfiehlt sich eine Provokation mit Nitroglycerin oder ein Belastungstest. Auch hier gelten $50 \mathrm{mmHg}$ als Grenzwert für die Obstruktion.

Mit der MRT lässt sich die myokardiale Masse exakt bestimmen und atypisch lokalisierte etwa spitzenbetonte Formen der HCM nachweisen; auch umschriebene Fibrosierungen (,late enhancement"), die als Risikofaktor für Arrhythmien und plötzlichen Herztod gelten, lassen sich im MRT erkennen. „Je mehr Fibrose, umso schlechter ist die Prognose“, so Frey.

\section{Genscreening: Ja oder nein?}

Der Stellenwert des Genscreenings wird kontrovers diskutiert. Dafür spricht, dass der HCM-Patient womöglich eindeutig identifiziert wird und eine Abgrenzung von anderen Formen der LV-Hypertrophie gelingt. Der Genotyp erlaubt eine Risikostratifizierung und für das Familienscreening ist diese genetische Diagnostik unverzichtbar. Andererseits ist nur bei 40\% der HCM-Patienten das kausale Gen bekannt. Auch gibt es Patienten mit mehreren Mutationen und die Genotyp-Phänotyp-Korrelation variiert sehr stark.

Und die Frequenz vieler Mutationen ist sehr niedrig. „Es gibt sogar ,Private Mutationen', die nur bei einem Patienten vorkommen", so Frey. Hinsichtlich der psychologischen Situation der Angehörigen könne der Gentest jedoch im Sinne eines "peace of mind" vorteilhaft sein, wenn die genetische Disposition ausgeschlossen wird.

\section{Nur bei Beschwerden therapieren}

Die gefürchtetste Komplikation der HCM ist der akute Herztod. Risikoprädiktoren sind die Obstruktion und der Nachweis von Fibrosierungen im MRT. „Eine elektrophysiologische Untersuchung zur Risikostratifizierung ist nicht sinnvoll", so Frey. Sie komme nur infrage, um supraventrikuläre Tachykardien aufzuspüren, die interventionell behandelt werden können. Grundsätzlich ist eine Therapie nur bei symptomatischen Patienten indiziert.

Medikamente der ersten Wahl bei HOCM und nicht-obstruktiver HCM sind nicht-vasodilatierende Betablocker: Man sollte die höchstmögliche Dosis anstreben. Sind Betablocker kontraindiziert oder werden sie nicht vertragen, empfiehlt sich Verapamil in hoher Dosis bis $480 \mathrm{mg}$ täglich. Eine neuere Studie mit Losartan (INHERIT) ergab keinen Effekt auf die myokardiale Muskelmasse; Komplikationen traten nicht auf.

Bei HCM-Patienten mit Vorhofflimmern ist unabhängig vom $\mathrm{CHA}_{2} \mathrm{DS}_{2}$-Score

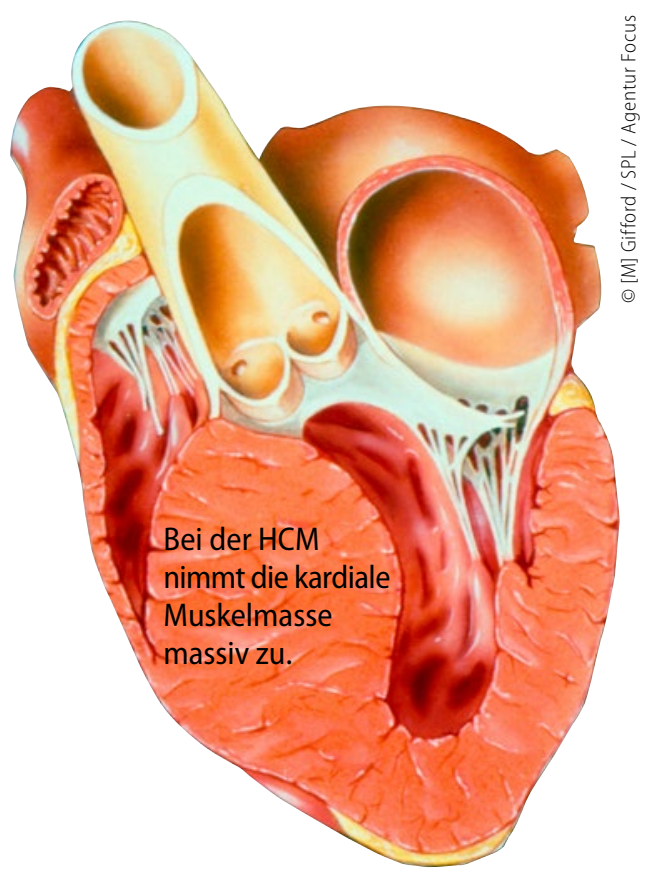

immer eine orale Antikoagulation indiziert. Bei Nachweis einer Obstruktion ist eine Septumreduktion indiziert, operativ oder mittels transkoronarer Ablation der Septumhypertrophie (TASH). Die Prognose kann dadurch langfristig verbessert werden, d. h. der Benefit zeigte sich in Studien erst in vier bis fünf Jahren.

\section{Wann ist ein ICD nötig?}

Die ICD-Implantation als Sekundärprävention nach vorausgegangener Reanimation oder anhaltender ventrikulärer Tachykardie (VT) ist unbestritten. Doch wie sieht es bei der Primärprävention aus? Bisherige Kriterien sind: positive Familienanamnese für den akuten Herztod, extreme Hypertrophie (> $30 \mathrm{~mm}$ ), vorausgegangene Synkopen, VT im Langzeit-EKG und Blutdruckabfall unter Belastung.

„Doch ein Teil der Patienten, die an einem akuten Herztod versterben, wird mit diesen Kriterien nicht erfasst", so Frey. Man habe daher nach besseren Prädiktoren gesucht und einen Risikokalkulator entwickelt. Wenn dieser ein Risiko für den akuten Herztod von unter 5\% angebe, sei ein ICD nicht indiziert. (Peter Stiefelhagen)

Cardiovasc 2015/4: 27, basierend auf: 36. BNK-Jahrestagung, 13.06.2015 in Berlin 